\title{
Probing the Higgs coupling to top quark in events with associated Higgs pro- duction with top-anti top pair and Higgs decay in two photons
}

\author{
Francesco Micheli ${ }^{1,2, a}$ \\ ${ }^{1}$ Sapienza, Universitá di Roma \\ ${ }^{2}$ INFN, Sezione di Roma
}

\begin{abstract}
We present here the results of the first search for the associated production of the Higgs boson with a $t \bar{t}$ pair, using the events where the Higgs boson decays to two photons in CMS. In order to maximize acceptance and sensitivity to such a small signal, we device two different sets of event selection criteria, optimized for leptonic and all-hadronic $t \bar{t}$ decays. In the absence of a significant excess over the background-only predictions, we proceed to set an observed (expected) 95\% confidence level upper limit on $t \bar{t} H$ production cross section times $B R(H \rightarrow \gamma \gamma)$ of 5.4 (5.3) times the Standard model value using $19.6 f b^{-1}$ of $8 \mathrm{TeV} p p$ collisions.
\end{abstract}

\section{Introduction}

Just after the discovery of a new boson, with a mass of 125 $\mathrm{GeV}$, compatible with the Standard Model (SM) Higgs boson, the upcoming challenge for experimenters is to measure its couplings to other SM particles. It is of great interest, in particular, to measure the coupling to the top quark, as its large mass may hint to the fact that it plays a special role in the context of electroweak symmetry breaking.

We investigate here the production of $t \bar{t}$ in events where the Higgs boson decays to photons. This final state allows to reconstruct the Higgs invariant mass with excellent resolution. The channel is limited from the extremely low branching ratio of Higgs boson decaying into two photons, i.e. $\mathrm{BR}(H \rightarrow \gamma \gamma)=0.0023$. In order to maximize acceptance to the signal, we will study the channels with the largest branching ratio for the top quark pair decays, i.e. the all-hadronic $t \bar{t} \rightarrow$ bqqbqq and leptonic $t \bar{t} \rightarrow$ blvbqq, $t \bar{t} \rightarrow$ blvblv decays, where 1 denotes either an electron or a muon.

The data used here was collected by Compact Muon Solenoid (CMS) [1] detector in 2012 using proton-proton collision data at a center of mass energy of $8 \mathrm{TeV}$ at the Large Hadron Collider (LHC), corresponding to an integrated luminosity of $19.5 \pm 0.9 \mathrm{fb}^{-1}$. The data has been recorded through the High Level Trigger (HLT) paths which rely on the presence of two high $p_{T}$ photons in the event, similarly to what is done in the untagged $H \rightarrow \gamma \gamma$ analysis[2].

The photon candidates are collected within the ECAL fiducial region $|\eta|<2.5$, excluding the barrel-endcap transition region $1.4442<|\eta|<1.566$. Isolation requirements are applied to photon candidates by looking at neighbouring particle candidates reconstructed with the particle-flow (PF) event reconstruction technique.

\footnotetext{
a e-mail: francesco.micheli@cern.ch
}

Jets are defined by clustering PF particle candidates with the anti-kT algorithm with a distance parameter of 0.5 . Jets are required to be inside the tracker acceptance ( $|\eta|$ less than 2.4) and are required to have $p_{T}>25 \mathrm{GeV}$.

To identify jets originating from the hadronization of bottom quarks, the Combined Secondary Vertex (CSV) btagging algorithm is employed. The algorithm identifies jets from b-hadron decays by identifying their displaced decay vertex.

Muons are measured with the combination of the tracker and the muon system, in the pseudorapidity range $|\eta|<2.4$. Electrons are detected as tracks in the tracker, pointing to energy clusters in the ECAL up to $|\eta|$ less equal than 2.5. Both muons and electrons are required to have a momentum transverse to the beam axis, $p_{T}$, greater than $20 \mathrm{GeV}$.

\section{2 ttH analysis in the diphoton channel}

The analysis strategy is to fit the diphoton mass distribution, where the diphoton spectrum sidebands will be used to fit the distribution of the backgrounds, and the presence of a signal around $125 \mathrm{GeV}$ is allowed compatibly with detector resolutions. To select events we can exploit the very characteristic final state of $t \bar{t} H$ events, with jets, b-jets and leptons in the final state, as can be seen in the diagram in Fig.1

Two sets of event selection criteria, which aim respectively at all-hadronic and semi-leptonic top pair decays in $t \bar{t} H$ events. The two channels benefit from the same photon selection, which requires the leading photon to have a transverse momentum greater than $60 \mathrm{GeV} \cdot m_{\gamma \gamma} / 120 \mathrm{GeV}$, and the subleading photon to have $p_{T}>25 \mathrm{GeV}$. The adoption of a variable threshold on the leading photon is aimed at reducing the kinematic turn-on. 


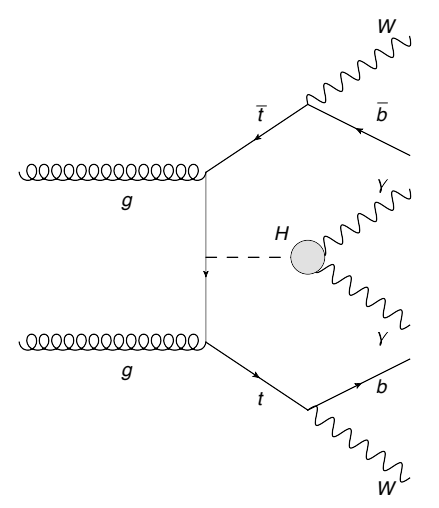

Figure 1. Feynman diagram for one of the LO diagrams of $t \bar{t} H$ at pp colliders, with Higgs decaying to photons.

In addition to this, both channels require the presence of at least one b-tagged jet; since we have always at least two real b-jets in the final state this request is $80 \%$ efficient on $t \bar{t} H$ signal. The hadronic channel is then defined by the requirement of at least four more jets in the event and no lepton, whereas the leptonic channel is defined by requiring at least one more jets in the event and at least one lepton (electron or muon).

The diphoton invariant mass spectra after full selections are shown in Fig.2, on the top for the hadronic channel and on the bottom for the leptonic channel. The data are fitted with a third- degree polynomial for the hadronic channel and with an exponential function for the leptonic one. The result of the fit is shown on the plot, together with the uncertainty bands corresponding to $68 \%$ and $95 \%$ probability. The expected contribution of a Standard Model Higgs boson is also shown as a blue histogram. The expected signal event yield after selections, is 0.65 for the hadronic channel and 0.44 for the leptonic one. The contribution of production modes other than $t \bar{t} H$ is minor, $13 \%$ in the hadronic channel and $3 \%$ in the leptonic one.

Currently the analysis is limited by statistics so the impact of systematics is very low. The main sources of systematic uncertainties are:

- Luminosity: The uncertainty on luminosity measurement is $4.5 \%$

- Photon reconstruction: The considered sources of systematic uncertainties for photon reconstruction are applied at single photon level and then propagated into the di-photon signal model using the MC. These contributions, related to photon identification, scale and resolution, are evaluated on $Z \rightarrow e^{+} e^{-}$and range from 1 to $2.5 \%$.

- Jet reconstruction: These uncertainties are related to jet energy scale and jet energy resolution. They are evaluated smearing the nominal values on MC and comparing event yields with nominal and smeared simulated samples. The value of these uncertainties is $\sim 1-2 \%$. A systematic uncertainty is also evaluated for btag efficiency smearing data/MC scale factors.
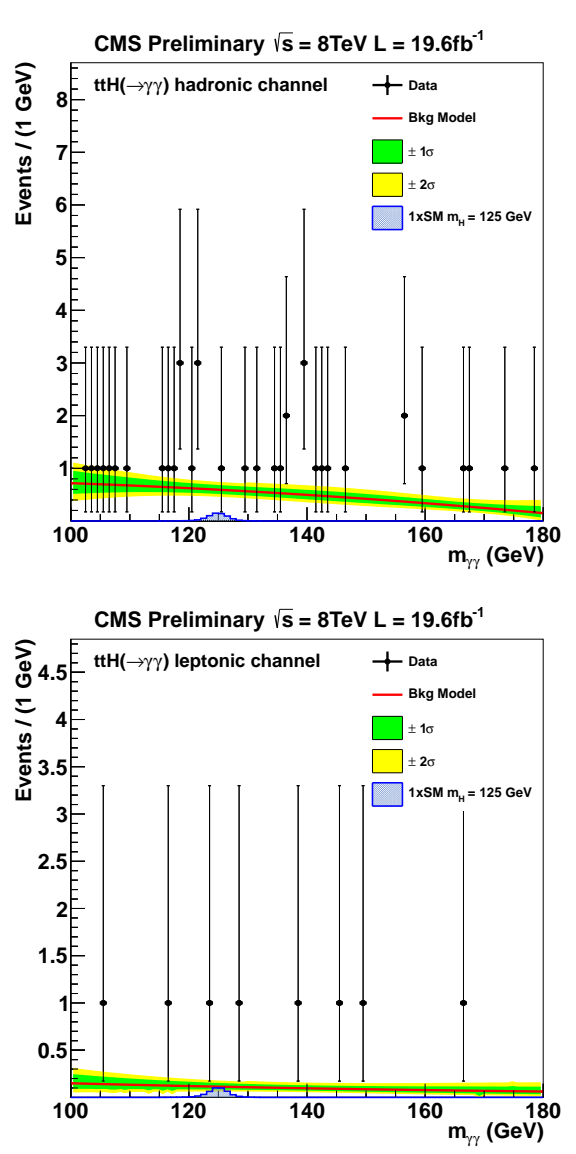

Figure 2. Diphoton invariant mass spectra after full selections for hadronic (top) and leptonic (bottom) channel

\section{Results}

By analyzing the invariant mass spectra of the two channels, an upper limit on the production cross section of Standard Model Higgs boson can be placed. The background model parametrisation is a second order polynomial for the hadronic channel and a simple exponential for the leptonic channel, whose shape parameters are floated during the likelihood fit. A 95\% confidence level exclusion limit on the signal strength modifier is evaluated using a modified frequentist approach, $C L_{S}$, taking the profile likelihood ratio as a test statistic.

The limits on the production cross section times branching ratio of a Higgs boson decaying to two photons relative to the SM expectation, for the combination of both channels, are shown in Figure 3. The expected limit is shown as a dotted black line, and the bands corresponding to $68 \%$ (green) and $95 \%$ (yellow) probability are added. The observed limit is signalled by the black solid line. In table 1 observed and expected limit for the different channel are reported. As can be seen, in the hadronic channel we observe a limit of 6.8 times the SM cross section (9.2 expected), while in the leptonic one we observe 10.7 times the SM cross section (8.0 expected). Combining the two channel we observe an upper limit of 5.4 the SM cross section. 
Using this analysis we can also fit $\mathrm{ttH}$ signal strenght $\mu_{t t H}$ normalized to the SM expectations. Using SM value for ggH production mode $\left(\mu_{g g H}=1\right)$ we obtain $\mu_{t t H}=$ $-0.2_{-1.9}^{+2.4}$. All the nuisance parameters are profiled during the fit.

\section{Conclusions}

The first search for production in the events where the Higgs boson decays to two photons is presented. In order to maximize acceptance and sensitivity to such a small signal, we device two different sets of event selection criteria, optimized for semileptonic and all-hadronic $t \bar{t}$ decays. In the absence of a significant excess over the backgroundonly predictions, we proceed to set an observed (expected) 95\% confidence level upper limit on the $t \bar{t} H$ production cross section times $B R(H \rightarrow \gamma \gamma)$ of 5.4 (5.3) times the
Standard Model value using $19.6 \mathrm{fb}^{-1}$ of $8 \mathrm{TeV}$ pp collisions. We can measure also ttH signal strenght and we obtain $\mu_{t t H}=-0.2_{-1.9}^{+2.4}$.

\section{References}

[1] CMS Collaboration, "The CMS experiment at the CERN LHC", Journal of Instrumentation, Vol.3 n.08 (2008)

[2] CMS Collaboration, "Updated measurements of the Higgs boson at $125 \mathrm{GeV}$ in the two photon decay channel", CMS Physics Analysis Summary CMS-PASHIG-13-001, (2013)

[3] CMS Collaboration, "Search for $t \bar{t} H$ production events with $H \rightarrow \gamma \gamma$ at $\sqrt{s}=8 \mathrm{TeV}$ collisions ", CMS Physics Analysis Summary CMS-PAS-HIG-13015, (2013)

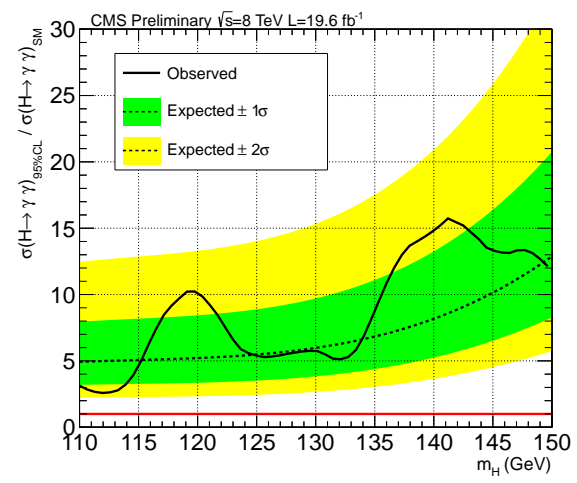

Figure 3. 95\% C.L. upper limit on $t \bar{t} H, H \rightarrow \gamma \gamma$ production combining results of the leptonic and hadronic channel, divided by the SM production cross section

Table 1. Expected and observed limit for hadronic, leptonic channel and the combination of the two.

\begin{tabular}{lccc}
\hline & Observed & Expected & Expected (no syst) \\
\hline Hadronic Channel & 6.8 & 9.2 & 8.8 \\
Leptonic Channel & 10.7 & 8.0 & 7.7 \\
Combined & 5.4 & 5.3 & 5.1 \\
\hline
\end{tabular}

